\title{
Interacciones y percepciones sociales con cocodrilo de río (Crocodylus acutus) en la costa sur de Jalisco, México
}

\author{
Social interactions with perceptions crocodile (Crocodylus acutus) \\ on the coast south of Jalisco, Mexico
}

\author{
Juan Luis Peña-Mondragón ${ }^{1}$, Andrés García ${ }^{2}$, Jorge H. Vega Rivera ${ }^{2}$, Alicia Castillo ${ }^{1}$ \\ Resumen
}

\begin{abstract}
Se documentó la percepción de los pobladores de dos comunidades rurales sobre el cocodrilo de río (Crocodylus acutus, Cuvier 1807) en la costa suroeste de Jalisco. Usando el método de entrevistas semi-estructuradas, se pudo documentar la percepción de los pobladores hacia la especie, donde se pudo evidenciar una negativa aceptación, porque se considera como peligrosa por el $85 \%$ de los entrevistados, justificado por el posible ataque a personas y ganado; otro resultado importante es que los pobladores perciben que las actividades de protección, ha incrementado el tamaño de la población de los cocodrilos. Las actitudes de rechazo llevan a proponer la eliminación de los cocodrilos o que los propietarios de la Reserva de la Biosfera Chamela-Cuixmala los cuiden o encierren. El 55\% de los encuestados mencionaron el aprovechamiento de los animales como una alternativa. Este estudio puede contribuir a la formulación de una estrategia que promueva la conservación de la especies en su hábitat natural, a través de motivar cambios en las percepciones, actitudes y valoraciones de los pobladores que comparten el hábitat con los cocodrilos. Se propone llevar a cabo acciones de educación ambiental que apoyen estos cambios, a la vez que se difunda información útil para la protección de las poblaciones humanas de posibles ataques.
\end{abstract}

Palabras clave: Chamela-Cuixmala, Conflicto humano-vida silvestre, Conservación, Crocodylia.

\begin{abstract}
It was documented the perception towards river crocodiles (Crocodylus acutus, Cuvier 1807) of two rural communities in the southwest Jalisco coast. Through semi-structured interviews, it was found that the perception towards the species is negative; $85 \%$ of interviewees considered it a dangerous animal because it attacks people and cattle. Results show that inhabitants perceive that due to protection activities, the crocodiles' population size has increased. Attitudes of rejection lead to propose the elimination of crocodiles or that the owners of the Chamela-Cuixmala Biosphere Reserve look after them or lock them in. Some people mentioned the use of the animals as an alternative (55\%). This study may contribute to the formulation of a strategy that promotes the conservation of the species through motivating changes in the perceptions, attitudes and valuation of the people that share the habitat with crocodiles. Environmental education actions are proposed that support these changes, at the same time that useful information is disseminated for the protection of people from possible attacks, as well as to evaluate possible economic uses of the species.
\end{abstract}

Keywords: Chamela-Cuixmala, Conflict human wildlife, Conservation, Crocodylia.

\section{Introducción}

Es cada vez más reconocido que el éxito o fracaso de los programas de conservación de cualquier especie depende, en gran medida, de las percepciones y actitudes que tienen los pobladores con la especie con quien comparte un hábitat (Woodroffe et al. 2005). En el caso de los animales carnívoros que

1 Laboratorio Comunicación para el Manejo de Ecosistemas, Centro de Investigaciones en Ecosistemas, Universidad Nacional Autónoma de México Campus Morelia, Morelia, México. e-mail: jlpena@cieco.unam.mx castillo@cieco.unam.mx

2 Estación de Biología, Instituto de Biología, Universidad Nacional Autónoma de México, Chamela, México. e-mail: chanoc@ib.unam.mx jhvega@ib.unam.mx

Fecha recepción: Enero 26, $2013 \quad$ Fecha aprobación: Marzo 15, 2013 
suelen causar daños tales como depredar ganado o amenazar las vidas humanas, es fundamental entender cómo se percibe a una especie que las ideas y visiones que se tengan sobre ésta determinarán las actitudes y acciones hacia ella (Lucherini y Merino 2008). En México se ha puesto particular atención a los conflictos causados por grandes felinos tales como pumas y jaguares, o por animales como los osos negros que generan daños en el patrimonio de los pobladores rurales (Peña-Mondragón 2011). Una especie que causa conflictos y para la que no existen estudios en este sentido es el cocodrilo de río Crocodylus acutus. El presente trabajo es una primera aproximación a entender este conflicto a partir de conocer las percepciones y experiencias de pobladores rurales en la región de la costa sur de Jalisco. El concepto de percepciones ambientales utilizado en este trabajo se tomó principalmente de Lazos y Paré (2000) quienes proponen que las percepciones se entienden como el sistema de creencias, actitudes y valoraciones que hacen los individuos de su entorno. Estas visiones del ambiente están determinadas tanto por la experiencia personal, como por la interacción social y los procesos históricos, culturales y políticos de un grupo social (Durand 2008). Se reconoce que las formas de percibir el ambiente influyen en las acciones de las personas y consecuentemente documentarlas es de gran utilidad para la formulación de intervenciones que busquen transformar las relaciones sociedad-naturaleza.

En la zona de estudio, la Universidad Nacional Autónoma de México tiene, desde 1971, una estación de investigación científica (Estación de Biología Chamela) que forma parte de la Reserva de la Biosfera Chamela-Cuixmala (RBChCx) desde 1993 (Diario Oficial de la Federación 1993). El río Cuitzmala delimita la parte sur de esta reserva y en su desembocadura, existen poblaciones de C. acutus que se encuentran bajo protección de la reserva. De acuerdo con García et al. (2010), a finales de 1980, la población de cocodrilos se estimaba en solo 35 individuos. Gracias a los esfuerzos de conservación de la Fundación Ecológica de Cuixmala AC (FEC) la población tuvo una recuperación muy favorable y para el año 2003, se contabilizaron 260 individuos y 440 nidos lo que les permitió a estos autores considerar la estabilización de la población. Con base en lo anterior, el objetivo de este trabajo fue documen- tar las experiencias y visiones de pobladores rurales en relación con el cocodrilo de río, con la finalidad de contribuir tanto al manejo de la especie como al diseño de estrategias de comunicación y educación ambiental que promuevan su conservación a la vez que se eviten daños a los habitantes locales.

\section{Metodología}

Área de estudio. Se seleccionaron las localidades de Emiliano Zapata y Francisco Villa en la costa sur de Jalisco (coordenadas de referencia: $19^{\circ} 23^{\prime} 5.88^{\prime \prime} \mathrm{N}$ y $\left.104^{\circ} 57^{\prime} 57.09^{\prime \prime} \mathrm{O}\right)$ debido a que colindan con la Reserva de la Biosfera ChamelaCuixmala en la desembocadura del río Cuitzmala en donde habita la población de cocodrilos, los cuales salen y entran de la zona de reserva a través de ríos y arroyos que comparten con los asentamientos humanos. Emiliano Zapata es un ejido [forma de propiedad que combina la posesión de terrenos individuales con propiedad comunal (Warman 2000), y Francisco Villa es una localidad de avecindados (personas que no poseen tierras)] localizado en el mismo núcleo urbano que Emiliano Zapata (Figura 1). Ambas localidades se fundaron en la década de 1960 (Castillo et al. 2005) y se caracterizan socio-económicamente por tener niveles bajos de bienestar, tasas elevadas de analfabetismo, altos índices de migración, un déficit en acceso a infraestructura y servicios básicos, además de poco acceso a fuentes continuas de empleo [Ceballos et al. 1999, Secretaría de Medio Ambiente para el Desarrollo Sustentable (SEMADES) 1999].

Métodos. Para la colecta de datos se utilizó una combinación de los métodos de observación participante (Tarrés 2004) y la entrevista semi-estructurada (Robson 1993). A través de la observación participante se estableció contacto y se logró el acceso a las comunidades de estudio. La entrevista semi-estructurada consiste en conversaciones con las personas usando preguntas abiertas sobre temas específicos (Vela 2001). Se entrevistaron un total de 22 personas entre 28 y 81 años. Para seleccionar a las personas y determinar la muestra se usó el método «bola de nieve» a través del cual se identificaron informantes clave que son aquellos individuos que poseen la información que responde a los objetivos de investigación (Sierra 1998), en este caso, aquellas personas que tuvieron alguna interacción con 
cocodrilos. Estos informantes dieron referencias de otros informantes y el tamaño de muestra se determinó cuando las respuestas obtenidas resultaron similares, determinándose así la saturación de datos (SánchezSerrano 2004). La información brindada en las entrevistas fue capturada por escrito (Anexo 1).

De acuerdo con los procedimientos establecidos en la investigación interpretativista, la información se analizó mediante la lectura línea por línea, y de acuerdo con las ideas encontradas en las respuestas, se construyeron las categorías (Strauss 1995). Para estas categorías se obtuvieron las frecuencias que se presentan en la sección de resultados donde se utilizan porcentajes como indicadores del número de personas que sostienen una determinada idea. Debido a que las preguntas fueron abiertas y no se dieron respuestas pre-establecidas, no es posible obtener $100 \%$ al sumar los porcentajes encontrados para cada categoría aunque, en ocasiones, las respuestas sean excluyentes.

\section{Resultados}

De los resultados obtenidos, cabe destacar que $85 \%$ de los entrevistados perciben a los cocodrilos como peligrosos, mientras que un mismo porcentaje mencionó que los cocodrilos no le gustan (no necesariamente las mismas personas), aunque solo 5 (22\%) de los entrevistados informó haber sido atacado y $3 \%$ de los entrevistados mencionó ataques a ganado por parte de los cocodrilos (Figuras 2 y 3 ). Es importante señalar también que $50 \%$ de los entrevistados piensa que los cocodrilos fueron introducidos por la Fundación Ecológica Cuixmala, la dueña de la mayor proporción de los terrenos que forman la $\mathrm{RBChCx}$. El 50\% de los entrevistados desconoce cuál es el papel de los cocodrilos en la naturaleza. Respecto a la utilidad de los cocodrilos, $55 \%$ reconocieron un uso peletero (aprovechamiento de la piel para fabricar prendas de vestir como cinturones, bolsos, zapatos, entre otros), $36 \%$ la obtención de carne y surgieron ideas también sobre su existencia para asustar o perjudicar a las personas. Un 76\% indicó que los cocodrilos deben removerse del área. Finalmente fue interesante documentar al menos cinco casos de ataques en un lapso de 13 años y un aspecto importante es que los entrevistados perciben los ataques como provocados por los mismos involucrados de forma accidental o por descuido y no por agresividad de la especie. Los cinco ataques documentados fueron en varones entre 28 y 42 años, atacados por cocodrilos mientras desarrollaban sus actividades cotidianas; los afectados mencionaron que se encontraban pescando mientras fueron atacados por los cocodrilos, pero a pesar de lo anterior reconocen que pudieron haber evitado el ataque siendo más cuidadosos.

\section{Discusión}

La recuperación del cocodrilo de río (Crocodilus acutus) en las inmediaciones de la $\mathrm{RBChCx}$, es un éxito desde el punto de vista biológico (García et al. 2010). Sin embargo, esta situación ha originado un conflicto con las comunidades humanas que habitan la zona cercana a los humedales en donde se encuentra la especie y que compromete su conservación a largo plazo. Los pobladores reconocen que las poblaciones de cocodrilo van en aumento tal y como ha sido confirmado por los monitoreos poblacionales de la especie (García et al. 2010), lo que ha derivado en un mayor número de encuentros entre los pobladores y los cocodrilos. Este es un patrón que también se ha presentado en el estado de Florida en Estados Unidos de América, en dónde las poblaciones de cocodrilo también han aumentado y los pobladores entienden que hay un mayor riesgo ante esta situación (Smithem 2005).

A lo largo de la costa de Jalisco la relación entre los grupos humanos y los cocodrilos incluye ataques a las personas. En los últimos 52 años hay documentados al menos 35 casos de ataques a humanos por cocodrilos incluyendo los reportados en este trabajo (Cupul-Magaña et al. 2010). Este número alto de ataques ha provocado un recrudecimiento y percepción negativa hacia los cocodrilos, como ocurre en otros sitios donde hay presencia de cocodrilos (Smithem 2005, Hernández et al. 2006, Wallace et al. 2012).

Es interesante la idea de que los cocodrilos fueron introducidos o que no había tantos hasta que «los de Cuixmala (en referencia a la FEC) los trajeron». $\mathrm{Al}$ respecto hay poca información que sugiriera la introducción de ejemplares, a diferencia de los estados vecinos Colima y Michoacán que han introducido en Unidades de Manejo y Aprovechamiento 
(UMA: figura legal de aprovechamiento cinegético en México) ejemplares incluso de especies distintas a las del lugar (C. moreletti) (Hernández et al. 2006). Los pobladores piden que se haga algo con los cocodrilos, pues consideran que no cumplen una función benéfica; desde su punto de vista sólo sirven para causar daños y «cuidar las tierras de los ricos». Este tipo de percepciones están presentes en otras zonas de la distribución de cocodrilos, tales como Colombia, en donde los pobladores piensan que el aumento de las poblaciones de estos animales sólo produce impactos negativos (Balaguera-Reina y GonzálezMaya 2010).

En algunos casos los entrevistados comentaron sobre la posibilidad de aprovechar la especie para carne y peletería, cuestión que reconocen García et al. (2010) y que permitiría la obtención de beneficios por los pobladores locales como se señala en el Plan de Manejo de la RBChCx (Ceballos et al. 1999). Asimismo, puede reconocerse también como una vía para el mantenimiento de las poblaciones del cocodrilo de río (Hernández et al. 2006). Por otro lado, existe en la región, desde hace más de 10 años, un ejido que lleva a cabo un programa de ecoturismo con cocodrilos, lo cual podría constituir otra alternativa de aprovechamiento.

Cabe señalar finalmente que para consolidar la conservación de la especie es indispensable, además, llevar a cabo un programa permanente de educación ambiental a través del cual se promuevan actitudes y valores positivos hacia esta especie, a la vez que se difunda información útil para la protección de las poblaciones humanas de posibles ataques (Hernández et al. 2006). Sumado a lo anterior es necesario continuar con el monitoreo poblacional de la especie, así como documentando el estado poblacional de sus presas y caracterizar las actividades y zonas de pesca que permitan generar un programa preventivo y de sensibilización con los pescadores y pobladores. Los pobladores reconocen que los ataques a las personas pudieron haberse evitado, pero al igual que otros ataques registrados en la costa de Jalisco y en Centroamérica, las prácticas pesqueras fomentan o pueden disparar el riesgo de ataque (Hernández et al. 2006). Puede decirse que estos encuentros seguirán aumentando debido a los cambios en las áreas naturales para el desarrollo de actividades pesqueras, acuicultura y de desarrollo agropecuario producien- do la fragmentación de los hábitats de numerosas especies (Hernández et al. 2006). No obstante, estos problemas pueden resolverse a través del dialogo entre sectores, en este caso entre los administradores de la $\mathrm{RBChCx}$ y los pobladores locales y proveyendo información útil a través de un programa educativo.

\section{Conclusiones}

Las poblaciones de cocodrilos en la región de estudio han aumentado como consecuencia del éxito de los programas de conservación llevados a cabo por la Reserva de la Biosfera Chamela-Cuixmala. Sin embargo, los resultados de este trabajo exploratorio muestran que a la par se ha generado una actitud negativa hacia la especie por los pobladores locales. Asimismo, se obtuvo información sobre ataques a personas y animales domésticos. Es indispensable llevar a cabo acciones de educación ambiental que den a conocer a la población la importancia ecológica de la especie, para lograr modificar las percepciones actuales y proveer información para la prevención de ataques. Aunado a lo anterior, promover programas de aprovechamiento de la especie (sugerido en la propia literatura científica) donde la población local pueda participar de beneficios económicos, sería un buen complemento para mitigar y resolver el conflicto presente entre las poblaciones de cocodrilos y los grupos humanos con los que comparte el hábitat.

\section{Agradecimientos}

El primer autor agradece al Consejo Nacional de Ciencia y Tecnología (CONACYT) por la beca de posgrado otorgada (333128/234322), y al financiamiento de Fondo Mixto CONACYT-Jalisco (99050), al Centro de Investigaciones en Ecosistemas y al Programa de Posgrado en Ciencias Biológicas de la Universidad Nacional Autónoma de México. Agradecemos profundamente a cada una de las personas en las comunidades que aceptaron conversar con nosotros. Agradecemos a dos revisores anónimos por las observaciones que ayudaron a mejorar el documento. Agradecemos también el apoyo recibido por la Estación de Biología Chamela UNAM, así como el apoyo técnico de H. Ferreira, A. López y A. Valencia. 


\section{Literatura citada}

Balaguera-Reina SA, González-Maya JF. 2010. Percepciones, conocimientos y relaciones entre los Crocodylia y poblaciones humanas en la Vía Parque Isla de Salamanca y su zona de amortiguamiento, Caribe colombiano. Rev Latinoam Conserv. 1: 53-63.

Castillo A, Magaña MA, Pujadas A, Martínez L, Godínez C. 2005. Understanding rural people interaction with ecosystems: a case study in a tropical dry forest of Mexico. Ecosystems. 8: 630-43.

Cupul-Magaña FG, Rubio-Delgado A, Reyes-Núñez C, TorresCampos E, Solís-Pecero LA. 2010. Ataques de cocodrilo de río (Crocodylus acutus) en Puerto Vallarta, Jalisco, México: presentación de cinco casos. Cuad Med Forense. 16: $153-60$.

Diario Oficial de la Federación. 1993. Decreto por el que se declara área natural protegida con el carácter de reserva de la biosfera, la región conocida como ChamelaCuixmala, ubicada en el Municipio de La Huerta, Jal. 30 de diciembre de 1993, México.

Durand L. 2008. De las percepciones a las perspectivas ambientales: una reflexión teórica sobre la antropología y la temática ambiental. Nueva Antropología. Rev Cien Soc. 68: 75-87.

García A, Valtierra-Azotla M, Cuarón A, Ceballos G. 2010. Tendencias poblaciones de Crocodylus acutus en condiciones de protección en la reserva de la biosfera Chamela-Cuixmala, Jalisco, México. Rev Latinoam Biodivers. 1: 52-62.

Hernández-Hurtado H, García de Quevedo Machain R, Hernández-Hurtado P, Jiménez-Quiroz MC, Espino-Barr E. 2006. Los cocodrilos de la costa Pacífico occidental (Michoacán, Colima y Jalisco) de México. En: JiménezQuiroz MC, Espino-Barr E. (Eds). Los recursos pesqueros y acuícolas de Jalisco, Colima y Michoacán. p. 375-89. México, DF: Secretaría de Agricultura, Ganadería, Desarrollo Rural, Pesca y Alimentación.

Lazos E, Paré L. 2000. Miradas indígenas sobre una naturaleza «entristecida»: percepciones del deterioro ambiental entre nahuas del sur de Veracruz. México, DF: Plaza y Valdés. $220 \mathrm{pp}$.

Lucherini M, Merino MJ. 2008. Perceptions of human-carnivore conflicts in the high Andes of Argentina. Mountain Res Develop. 28: 81-5.
Peña-Mondragón JL. 2011. Daños económicos al ganado y percepciones sociales sobre el jaguar (Panthera onca veraecrucis Nelson and Goldman, 1933) en la Gran Sierra plegada, Nuevo León, México. Tesis Maestría. Morelia: Centro de Investigaciones en Ecosistemas, Universidad Nacional Autónoma de México128 pp.

Robson C.1993. Real World Reseach. A Resource for Social Scientists and Practitioner-Researchers. Oxford: Blackwell Publisher. 599 pp.

Sánchez-Serrano R. 2004. La observación participante como escenario y configuración de la diversidad de significados. En: Tarrés ML (Coordinadora). Observar, escuchar y comprender. Sobre la tradición cualitativa en la investigación social. MÁ Porrúa (Eds). México, DF: FLACSO, El Colegio de México. p. 97-130.

Secretaría de Medio Ambiente para el Desarrollo Sustentable (SEMADES) 1999. Ordenamiento Ecológico de la región costa del estado de Jalisco. URL disponible en: http:// www.semades.gob.mx/oe. México.

Sierra F. 1998. Función y sentido de la entrevista cualitativa en investigación social. En: Galindo CJ (eds). Técnicas de investigación en sociedad, cultura y comunicación. México; DF: Addison Wasley Longman. p. 277-345.

Smithem JL. 2005. Risk perceptions of and acceptance capacity for the American crocodile (Crocodylus acutus) in south Florida. Miami: University of Florida. 77 pp

Strauss AL. 1995. Qualitative analysis for social scientists. Cambridge: Cambridge University Press. 377 pp.

Tarrés ML. 2004. Lo cualitativo como tradición. En: Tarrés ML (Coordinadora). Observar, escuchar y comprender. Sobre la tradición cualitativa en investigación social. MÁ Porrúa. México, DF: FLACSO, El Colegio de México. p. 35-60.

Vela PF. 2001. Un acto metodológico básico de la investigación social: la entrevista cualitativa. En: En: Tarrés ML (Coordinadora). Observar, escuchar y comprender. Sobre la tradición cualitativa en investigación social. MÁ Porrúa. México, DF: FLACSO, El Colegio de México. p. 63-95.

Wallace KM, Leslie AJ, Coulson T. 2012. Living with predators: a focus on the issues of human-crocodile conflict within the lower Zambezi valley. Wildlife Research 38: 747-55.

Warman A. 2001. El campo mexicano en el siglo XX. México DF: Fondo de Cultura Económica. 262 pp.

Woodroffe R, Thirgood S, Rabinowitz A. 2005. The impact of human-wildlife conflict on natural systems. Conservat Biol. 9: 1-11. 